Psychosis, 2014

http://dx.doi.org/10.1080/17522439.2014.913073

Routledge

Taylor \& Francis Group

\title{
Using the back door: Metacognitive training for psychosis
}

\author{
Devvarta Kumar $^{\mathrm{a}, 1 *}$, Mahesh Menon ${ }^{\mathrm{b}, \mathrm{c}, 1}$, Steffen Moritz ${ }^{\mathrm{d}}$ and Todd S. Woodward ${ }^{\mathrm{b}, \mathrm{e}}$ \\ ${ }^{a}$ Department of Clinical Psychology, National Institute of Mental Health and Neuro Sciences, \\ Bangalore, India; ${ }^{b}$ Department of Psychiatry, University of British Columbia, Vancouver, \\ British Columbia, Canada; ${ }^{c}$ Vancouver Coastal Health Research Institute, Vancouver, \\ Canada; ${ }^{d}$ University Medical Center Hamburg-Eppendorf, Department of Psychiatry and \\ Psychotherapy, Martinistraße, Hamburg, Germany; ${ }^{e} B C$ Mental Health and Addictions \\ Research Institute, Vancouver, British Columbia, Canada
}

(Received 20 November 2013; accepted 4 April 2014)

\begin{abstract}
Delusions have traditionally been considered impervious to counter-arguments and thus not amenable to psychotherapy. However, a growing body of evidence from Cognitive Behavior Therapy for psychosis (CBT-p) has indicated that challenging the delusional beliefs may be effective in reducing their severity. Metacognitive Training/Therapy (MCT) for psychosis also targets delusions, using a back door approach by helping clients gain insight into the cognitive biases behind delusions, followed by attempts to plant the seeds of doubt, and weaken delusional beliefs. There are two variants of MCT, the group format MCT and the individual therapy format MCT (i.e. MCT+). The MCT intervention has three components: (a) normalization, (b) facilitating insight into the relationship between cognitive biases and delusions, and (c) sowing the seeds of doubt in delusional beliefs. Among these, the first two components are common to both MCT and MCT+, whereas the third is specific to MCT+. Initial findings about the effects of MCT in reducing the delusional convictions are encouraging. The present article elaborates on the theoretical background, process, clinical implications, empirical status, and the advantages and limitations of this intervention.
\end{abstract}

Keywords: metacognitive training; schizophrenia; CBT; psychosis; MCT

Pharmacological approaches have remained a mainstay of treatment for delusions since their introduction in the 1950s. However, there is growing realization among clinicians that pharmacological treatment has its limitations (Andrade, Radhakrishnan, \& Fernandes, 2012). Many individuals with psychosis have symptoms that respond partially or poorly to antipsychotics (Elkis, 2007), and relapse rates are high (Leucht, Arbter, Engel, Kissling, \& Davis, 2009). Antipsychotic medication may reduce delusional intensity; however, it is less likely to directly help in deconstruction of the delusional framework, which could have origins in one's life events, motivations, and cognitive biases (McKay, Langdon, \& Coltheart, 2005). Therefore, psychotherapeutic approaches that disassemble the scaffolding of the delusional framework itself may be required.

Phenomenologically, delusions have been considered impervious to counterarguments (Jaspers, 1963) and thus not amenable to psychotherapy. It is argued that

*Corresponding author. Email: devavrata.kumar@gmail.com

${ }^{1}$ The first two authors have equally contributed and therefore share first authorship

(C) 2014 Taylor \& Francis 
the imperviousness to counter-arguments may reflect a state of informational encapsulation of delusions (Jones, Delespaul, \& van Os, 2003). However, research showing the effectiveness of Cognitive Behavior Therapy for psychosis (CBT-p), which involves examining the delusional beliefs and facilitating the process of developing alternate perspectives for the delusional beliefs (Sivec \& Montesano, 2012; Tai \& Turkington, 2009), prove that delusions may be amenable to counter-arguments and challenges. In general, various cognitive explanatory models (of delusions) assume that irrational beliefs and misinterpretations may lead to delusions and, thus, attempts to correct these beliefs and interpretations can be useful (Pfammatter, Junghan, \& Brenner, 2006). Metacognitive Training (MCT) for individuals with psychosis is a novel psychotherapeutic technique which takes a "back door approach" to challenge and alter the framework of delusional beliefs (Moritz, \& Woodward, 2007; Moritz, Vitzthum, Randjbar, \& Woodward, 2010; Moritz et al., 2012). MCT's back door approach is grounded on the principle that prior to the attempts to challenge and alter the framework of the delusional beliefs, an individual should be helped in developing insight into the mechanisms of formation and maintenance of delusions. This counters the tendency to selectively attend to evidence that support the delusion (which could, in turn, reduce the chances of preferential assimilation of counter-evidence) thus strengthening the delusional beliefs. In this way, MCT paves the way for more objective evaluation of these beliefs.

In this article, we address the theoretical basis for MCT, the components of therapy (with clinical examples), and research supporting its efficacy. We then highlight its advantages and limitations. It is worth mentioning here that there are other MCT programs; for example, metacognitive therapy for anxiety and depression by Adrian Wells (Wells, 2009), which are fairly different in their approaches. The present review is limited to group and individualized MCT for psychosis developed by Steffen Moritz, Todd Woodward and colleagues (Moritz \& Woodward, 2007; Moritz et al., 2012).

\section{Theoretical background}

Deficits in social cognition (such as the "Theory of Mind") and the presence of certain cognitive biases (such as the "Jumping to Conclusions" (JTC), attributional biases, and Bias Against Disconfirmatory Evidence (BADE)) have been unequivocally reported in individuals with schizophrenia (Freeman, 2007; Garety \& Freeman, 1999; Garety \& Freeman, 2013; Moritz \& Woodward, 2006a; Woodward, Moritz, Cuttler, $\&$ Whitman, 2006). These deficits and biases make people vulnerable to misinterpretation of events, and maintain a rigid stance about them. For example, the tendency to arrive at a judgment or decision without gathering sufficient information allows for the formation of delusional ideas, as interpretations which are congruent with the individual's current emotional state (e.g. paranoia) might be considered likely without proper evaluation of those interpretations (Garety \& Freeman, 1999; Moritz \& Woodward, 2006a; Speechley, Whitman, \& Woodward, 2010). A related cognitive bias which is displayed frequently by individuals with schizophrenia, and which strengthens delusional beliefs, is the BADE, which refers to a tendency to minimize or disregard evidence that counter one's beliefs (Woodward, Moritz, Cutler, \& Whitman, 2006). Likewise, an individual's tendency to blame others for negative events in their life (Kinderman \& Bentall, 1997) reflects one-sided causal attribution for an event, known as the "attributional bias". This explanatory bias reduces self/ 
ideal discrepancies, but may trigger persecutory ideation (Bentall, Kinderman, \& Kaney, 1994).

Apart from these cognitive biases, errors in the form of misjudging others' emotions (especially the subtle emotions) and meta-memory deficits (particularly overconfidence in the accuracy of their memories), are commonly seen in schizophrenia (Moritz \& Woodward, 2006b). Research related to "Theory of Mind" (ToM) has underscored difficulties in understanding the emotions and mental states of others in individuals with schizophrenia (Lysaker et al., 2011; Pickup \& Frith, 2001). This may lead to a biased perception of intentions of others and cause increases in persecutory ideation.

There are indications that various cognitive biases may differ in their role in the formation and maintenance of delusions (So et al., 2010). MCT is based on the assumption that if individuals with schizophrenia increase their understanding regarding these cognitive errors and their possible role in the genesis and maintenance of delusional beliefs in a comprehensive manner, they can use this information to evaluate or alter their own delusional beliefs (Moritz \& Woodward, 2007; Moritz et al., 2010, 2012). Thus, while some modules of MCT highlight the need for caution and being flexible in decision-making, others emphasize why one should critically evaluate his/her memories before coming to conclusions about any past experience, the need for paying attention to the whole context before deciphering someone's emotion and on ways to improve mood and self-esteem.

MCT was initially developed as a group intervention consisting of a total of eight modules. In each module, the group is introduced to the targeted cognitive bias (e.g. JTC) and then, in a simple manner, explained the scientific findings with examples demonstrating how these errors can be associated with psychotic experiences. Following this, participants are given practice exercises highlighting how a given cognitive bias is formed and suggestions on how to counter them.

The group MCT has been supplemented with a variant (known as MCT+), which is an individual format, tailored to the individual's specific delusions (Moritz et al., 2010, 2012). MCT+ comprises a total of 10 therapy units. The first three units focus on the establishment of the therapeutic relationship, introduction to the concept of metacognition (and the metacognitive therapy) and developing an individual illness model. The next six units deal with common cognitive biases that may lead to the formation of delusional beliefs and the impact of psychotic symptoms on mood and self-esteem. The last unit targets relapse prevention and enhancement of the coping strategies. The uniqueness of MCT + is that it proceeds with the particular focus on metacognitive biases and this focus on metacognition aids the process of testing individual specific delusional beliefs through discussions, exercises, behavioral experiments and so forth. MCT+ also allows for some flexibility of session structure, to increase focus on certain modules if indicated, and reiterate, or explore specific areas of difficulty for each individual.

\section{Therapy process ${ }^{1}$}

Initially, the concept of "metacognition" needs to be introduced to the client as thinking about one's own thinking. It should be explained in a manner that facilitates understanding of the need for thinking about one's own beliefs. The intervention typically has the following three components: 
(a) Normalization

The crux of this component is to facilitate insight into the possibilities of errors in one's interpretation of events, and generally, to understand that "to err is human". The therapist first attempts to facilitate insight and metacognitive awareness about certain cognitive biases that can lead to faulty judgments about events, persons and situations. This is fostered by different examples relating to various issues, such as historical events, to explain how a one-sided causal attribution of an event can prevent an individual from understanding the broader context of that event, and how reaching conclusions without gathering enough information about an event may lead to incorrect inferences. For instance, in one of the therapy units on "decision-making", an example explains how urban legends and conspiracy theories emerge (e.g. the idea that Elvis faked his own death). These legends involve emotionally salient ideas and attention is selectively focused on certain details favoring those ideas (ignoring other details that may counter them). Also, the facts are explained out of context and, eventually, conspiracy theories emerge.

(b) Facilitating insight into the relationship between cognitive biases and delusions

The next component focuses on highlighting the possible links between an escalation of cognitive biases and delusions. A part of each module is devoted to discussing, in simple terms, each cognitive bias pertaining to the formation and maintenance of delusions, such as JTC, BADE, and so on. For example, the therapist could explain JTC in this manner: "We may make decisions without knowing the whole truth. These decisions may apply to simple everyday matters, for example, "what should I eat for lunch today?" or can concern complex social situations, related to our friends or environment in general. Could we think about some situations where hasty decisions can have dramatic consequences and others where it may be useful to not take too long making a decision?" With the help of discussion, exercises and homework assignments, the individual learns how a cognitive bias creates problems in making correct inferences about an event and may even foster psychotic episodes. Thus, after highlighting the fallibility of human cognition (the "normalization" process), this component of intervention intends to make the individual understand that if cognitive errors and biases persist they may be fertile ground for the genesis of delusions. After going through sessions on cognitive biases, a client diagnosed with Paranoid Schizophrenia (a single woman in her early 30s) summarized her ideas about quick and hasty decisions: "[I am able to] develop an open mind and broader perspectives - by trying to consider various possible angles before making a decision. [One should] be calm and take time before taking a decision".

(c) Sowing the seeds of doubt in delusional beliefs

In MCT+, in a gentle and non-confrontational manner, the individual is helped to evaluate his/her own beliefs in the light of new understandings about cognitive biases and their probable role in the formation and maintenance of delusional beliefs. This component of MCT + makes the second component of the training more concrete, personal, immediate, and experience-based. 
Delusions are not easily shaken and, therefore, individuals with psychosis may exhibit resistance in discussing their own delusional beliefs in the light of cognitive biases. However, efforts to make an individual understand that (s)he simply has to evaluate the information and is free to accept or reject the resulting explanations as they apply to specific beliefs, often prove fruitful. Once (s)he starts generating counter-evidence, this can lead to a kernel of doubt about the delusional beliefs, which may be strengthened if the process of questioning the thoughts and generating counter-evidences are gently but frequently encouraged. For instance, a client with delusions of reference summarized her ideas about quick and hasty decisions,

When I used to walk on the road and observed someone laughing, I used to think that he was laughing at me. But without any evidence, I now realize, that I cannot jump to conclusions that the person is laughing at me. He may be laughing for many reasons; for example, sharing a joke with someone.

Despite this, it is important to remember that some individuals are not forthcoming in relating their insights into cognitive biases with their own delusions. For instance, a client diagnosed with Paranoid Schizophrenia (a single woman in her late 20s), with delusions of control and persecution completed the sessions on cognitive biases and was encouraged to explore her thoughts in the background of her knowledge about these biases. However, she vehemently refused the idea that there was any role of the cognitive biases in the formation of her beliefs. She stated that certain physical sensations not observable to others were the evidence, and that she was not merely jumping to conclusions.

In such situations, using behavioral experiments (an integral part of $\mathrm{MCT}+$ ) often proves useful. Behavioral experiments give individuals an opportunity to test their beliefs and evaluate the plausibility of the alternative (non-delusional) explanations. For instance, a client with a diagnosis of Paranoid Schizophrenia (a single male in his late 20s) had the belief that people who crossed his path when he was walking could read his thoughts, and formed a negative impression of him, which he could decipher from their gestures. After discussing modules related to JTC and BADE, he started generating examples of how in day-to-day life people tend to reach to conclusions without proper evidence. However, he was reluctant in accepting a role of JTC in his beliefs. At this juncture, the therapist asked him if he could participate in an experiment in which he was requested to walk slowly on a footpath and carefully observe the gestures of 5-6 people who crossed him. He was then asked to watch people (who did not cross him) from a distance. He was asked to compare the gestures of these two groups of people and judge if the first group was very different in terms of gestures in comparison to the other group. On completing the exercise, he agreed that there was not much difference between the gestures of people who crossed his path from those who did not. This led him to consider the idea that he might have been over-interpreting the gestures of people crossing his path.

In order to facilitate generalization and sustenance of gains achieved during therapy, regular follow-up is important. This can help in evaluating whether the individual is utilizing what they have learned about counteracting the delusional beliefs.

\section{Clinical implications}

The primary clinical goal of MCT and MCT + is to allow people with psychosis to examine their beliefs and consider the possibility of cognitive biases and the 
presence of alternative explanations, thus assisting in altering the framework of delusions. As the emphasis of this approach is on facilitating insight into the cognitive biases and their possible links with the formation and maintenance of delusions, it is possible that they utilize this insight and generalize it to any such beliefs in future. Thus, it provides tools that may provide benefits post treatment, decreasing the chances of relapse.

\section{Research evidence}

Early empirical studies have suggested that MCT is effective (see Table 1). It has been found to reduce the delusional conviction and intensity of distress exerted by delusions, with findings typically in the range of medium effect sizes (reflecting MCT group effects over and above control group effects) (Aghotor, Pfueller, Moritz, Weisbrod, \& Roesch-Ely, 2010; Erawati et al., 2014; Favrod, Maire, Bardy, Pernier, \& Bonsack, 2011; Kumar et al., 2010; Moritz, Kerstan et al., 2011; Moritz, Veckenstedt, et al., 2011; Moritz et al., 2013). The improvements are also seen in interpersonal and psychosocial functioning domains (Moritz et al., 2010; Moritz, Kerstan et al., 2011; Naughton et al., 2012). Also, it is important to note that irrespective of overall change in psychopathology scores, reduction in the JTC bias has been reported across studies (Aghotor et al., 2010; Moritz, Kerstan et al., 2011; Moritz, Veckenstedt et al., 2011). Reduction in JTC is important, as it is considered a risk factor for positive symptoms of schizophrenia (Freeman, 2007). In a case study, MCT was also found to be effective in treating treatment resistant symptoms (Kumar et al., 2013). Results of studies with follow-up data indicate maintenance of the effects of MCT over time in terms of decreased severity and other dimensions of delusion (see Table 1; Favrod et al., 2013; Moritz et al., 2013).

\section{Advantages and limitations}

Although MCT (group and individual therapy formats) is a manualized treatment with specific guidelines and modules of sessions, it allows for much flexibility, and the clinician can adapt it according to the individual patient's needs (Moritz et al., 2012).

MCT has both individual and group formats. The group MCT has the advantage that the group members share their ideas about cognitive biases and the implications in psychosis, but specific delusions of any member need not be part of the discussion, facilitating openness and flow of ideas. On the other hand, individual therapy format $\mathrm{MCT}$ (the $\mathrm{MCT}+$ ) allows the client and therapist work collaboratively to evaluate the individual's specific delusions.

The modules are self-explanatory and a therapist can proceed in sessions easily. Moreover, the manual describes in detail how a therapist can establish a collaborative therapeutic relationship with the client, avoiding common traps that may lead to alliance rupture (Moritz et al., 2012). Thus, MCT can be carried out by a range of qualified mental health professionals and does not necessarily require much specialized training. However, given the nature of the content, it is advisable that the potential MCT therapist have a good understanding of psychosis, as the therapy is expected to lead to an in-depth discussion between the client and the therapist regarding various aspects of delusions. 


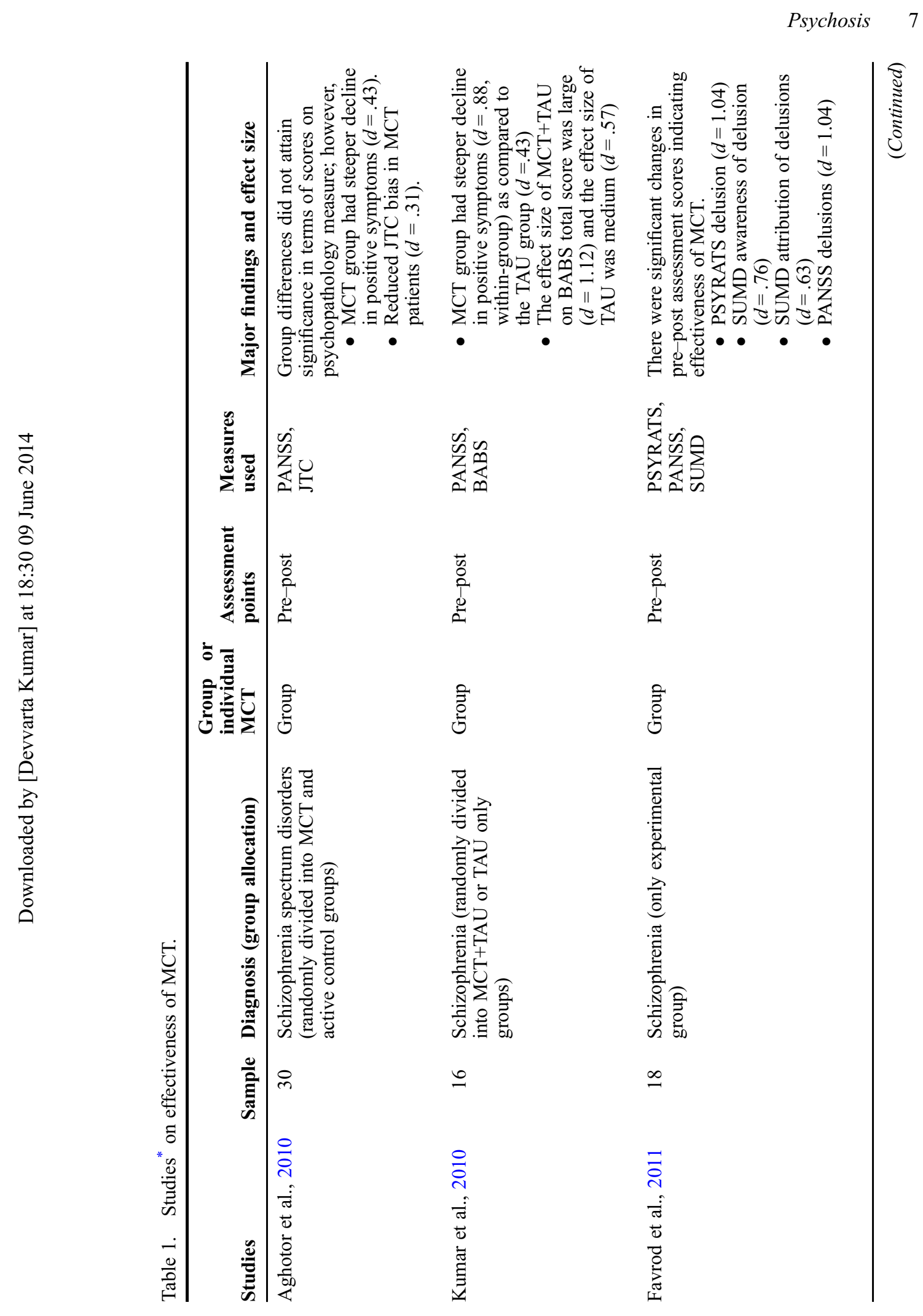




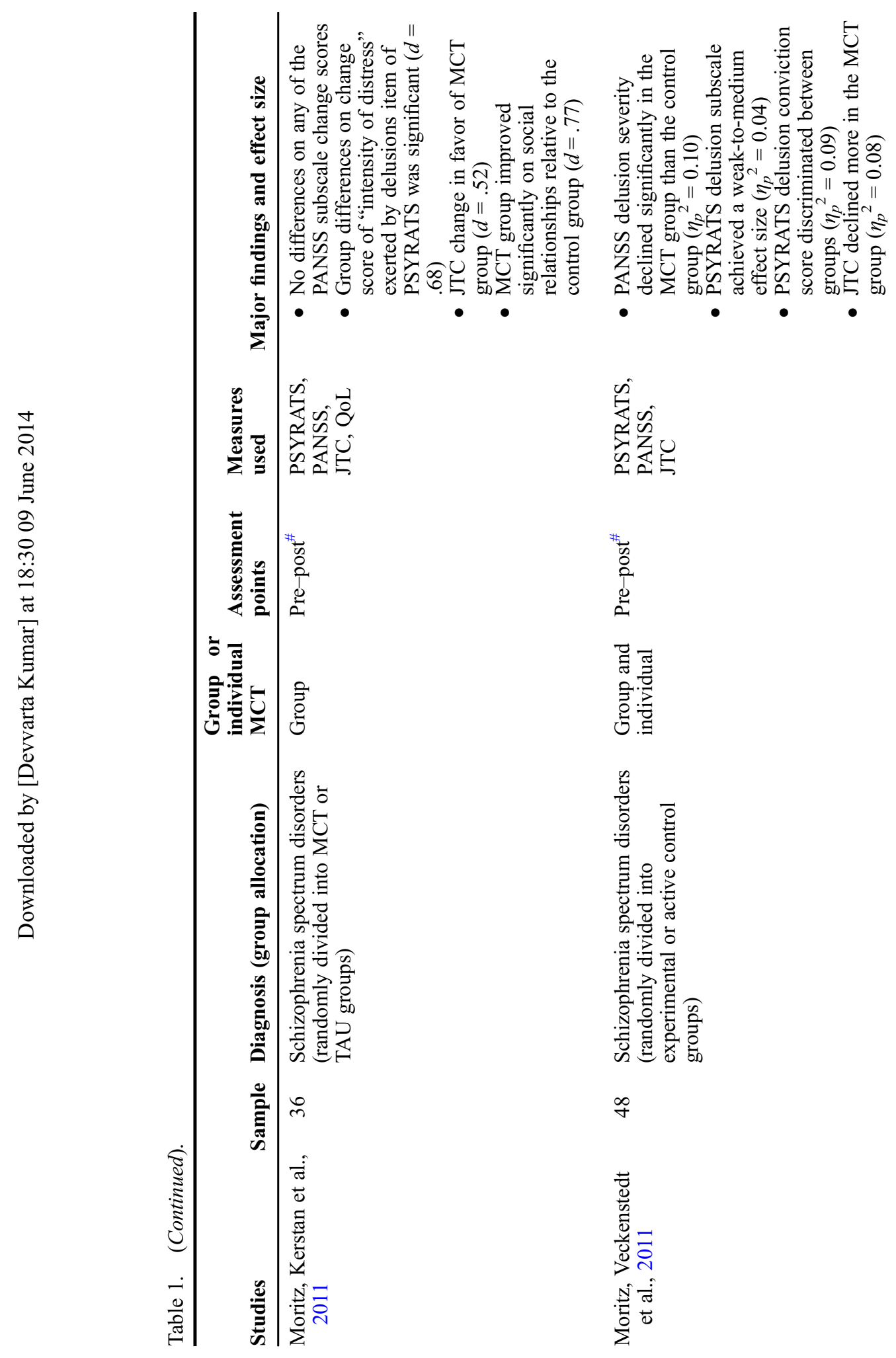


Psychosis 9
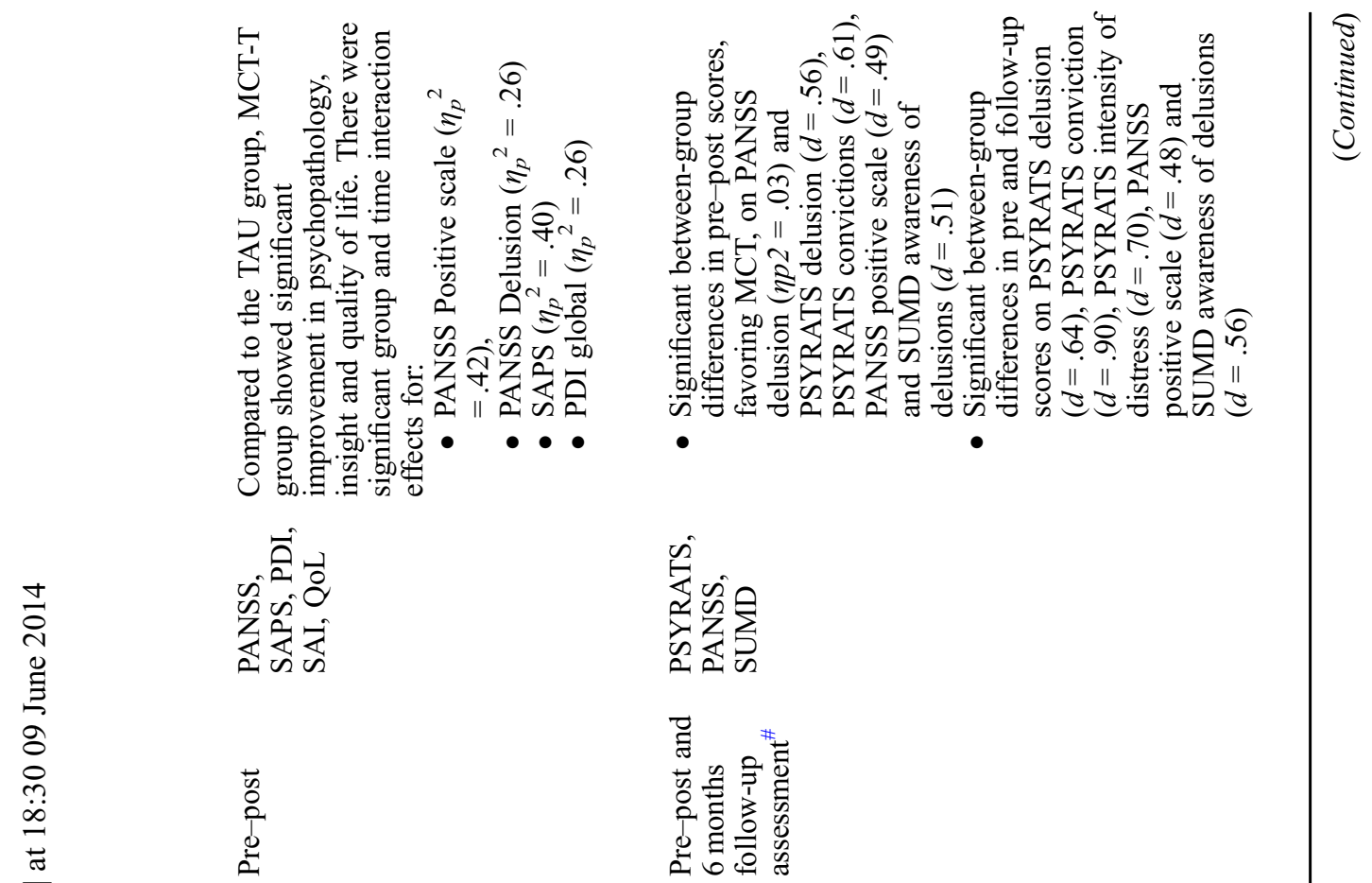

胥

ڤ̊ํำ
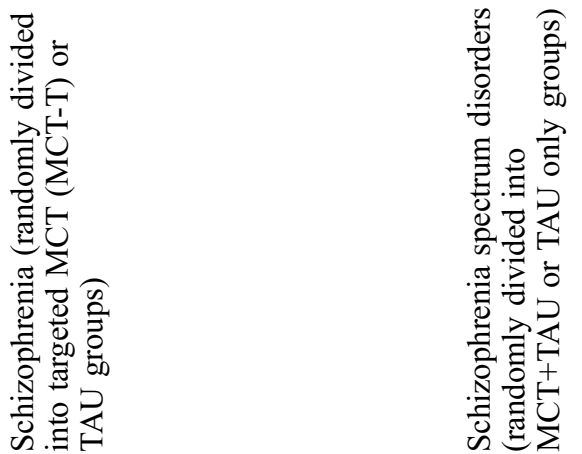

$\stackrel{\infty}{N}$

n

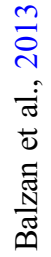

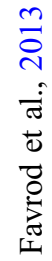




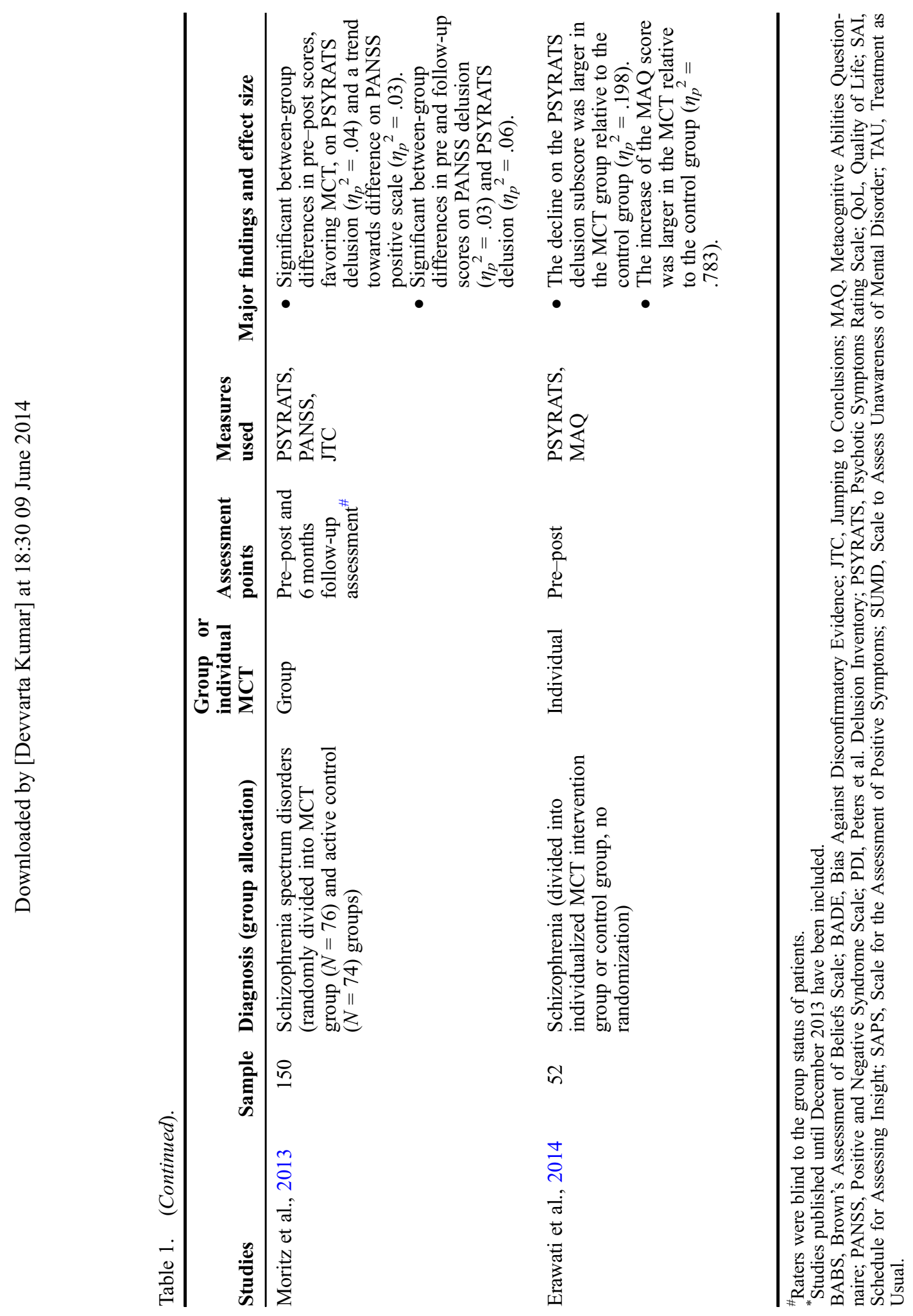


The MCT modules and manuals are freely downloadable (the group MCT modules have been translated in more than 30 languages and the MCT + is currently available in 7 languages: www.uke.de/mct_plus). The group format facilitates its use in clinics with limited resources.

MCT easily complements treatment using a CBT approach. In fact, the MCT+ is a blend of CBT for psychosis and MCT where initially a "back door" approach is used to instill insight into cognitive biases, followed by attempts, similar to CBT-p, to evaluate and challenge delusions specific to an individual.

MCT modules are presented in an easy to understand and interesting manner. Subjectively many individuals report that they had fun while going through this program and that they utilize the information learnt more often; for example, thinking about alternative explanations before judging any person or situation (Erawati et al., 2014; Moritz et al., 2013).

One limitation of MCT is that the stimuli used in the modules, although available in 30 languages, have not undergone much cross-cultural validation. It is important that the content, specifically the practice examples and stimuli, be evaluated for their cross-cultural validity. Likewise, studies are warranted to examine the moderators such as the quality of the client-therapist alliance, client characteristics to determine favorable or unfavorable outcomes of MCT and MCT + . Furthermore, studies are needed to see how group or individual therapy format MCTs compare to other psychosocial interventions.

\section{Conclusion}

MCT is a novel therapeutic approach and the initial empirical findings are encouraging. A structured approach, ease of use, and cost-effectiveness are a few advantages of this intervention. However, lack of cross-cultural validation of stimuli used in modules is a limitation. Future research is warranted to develop the MCT material and examine the effect of variables such as the nature of client-therapist alliance, client characteristics and so on that may determine favorable or unfavorable outcomes and explore the role of MCT in early intervention in psychosis.

\section{Acknowledgment}

We thank our patients for their willingness to engage in the psychotherapeutic process.

\section{Note}

1. All the patients mentioned in this article were also receiving pharmacological treatment. The first author was the therapist for all patients described in the article.

\section{References}

Aghotor, J., Pfueller, U., Moritz, S., Weisbrod, M., \& Roesch-Ely, D. (2010). Metacognitive training for patients with schizophrenia (MCT): Feasibility and preliminary evidence for its efficacy. Journal of Behavior Therapy and Experimental Psychiatry, 41, 207-211.

Andrade, C., Radhakrishnan, R., \& Fernandes, P.P. (2012). Psychopharmacology of schizophrenia: Future looks bleak. Mens Sana Monograph, 10, 4-12.

Balzan, R.P., Delfabbro, P.H., Galletly, C.A., \& Woodward, T.S. (2013). Metacognitive training for patients with schizophrenia: Preliminary evidence for a targeted, single-module programme. Australian and New Zealand Journal of Psychiatry. doi:10.1177/ 0004867413508451 
Bentall, R.P., Kinderman, P., \& Kaney, S. (1994). The self, attributional processes and abnormal beliefs: Towards a model of persecutory delusions. Behavior Research and Therapy, $32,331-341$.

Elkis, H. (2007). Treatment-resistant schizophrenia. Psychiatric Clinics of North America, 30, 511-533.

Erawati, E., Keliat, B.A., \& Helena, N. (2014). The influence of metacognitive training on delusion severity and metacognitive ability in schizophrenia. Journal of Psychiatric and Mental Health Nursing. doi:10.1111/jpm.12130

Favrod, J., Maire, A., Bardy, S., Pernier, S., \& Bonsack, C. (2011). Improving insight into delusions: A pilot study of metacognitive training for patients with schizophrenia. Journal of Advanced Nursing, 67, 401-407.

Favrod, J., Rexhaj, S., Bardy, S., Ferrari, P., Hayoz, C., Moritz, S., Conus, P., \& Bonsack, C. (2013). Sustained antipsychotic effect of metacognitive training in psychosis: A randomized-controlled study. European Psychiatry: http://dx.doi.org/10.1016/j.eurpsy.2013.08. 003

Freeman, D. (2007). Suspicious minds: The psychology of persecutory delusions. Clinical Psychology Review, 27, 425-457.

Garety, P.A., \& Freeman, D. (1999). Cognitive approaches to delusions: A critical review of theories and evidence. British Journal of Clinical Psychology, 38, 113-154.

Garety, P.A., \& Freeman, D. (2013). The past and future of delusions research: From the inexplicable to the treatable. British Journal of Psychiatry, 203, 327-333.

Jaspers, K. (1963). General psychopathology. (J. Hoenig \& M.W. Hamilton, Trans). Manchester, NH: Manchester University Press.

Jones, H., Delespaul, P., \& van Os, J. (2003). Jaspers was right after all - delusions are distinct from normal beliefs. British Journal of Psychiatry, 183, 285-286.

Kinderman, P., \& Bentall, R.P. (1997). Causal attributions in paranoia and depression: Internal, personal, and situational attributions for negative events. Journal of Abnormal Psychology, 106, 341-345.

Kumar, D., Rao, M.N., Dhanya, R., Venkatasubramanian, G., Vermabally, S., \& Gangadhar, B.N. (2013). Metacognitive training for delusion in treatment resistant schizophrenia: A case report. Clinical Schizophrenia and Related Psychoses. doi:10.3371/ CSRP.KURA.031513

Kumar, D., Zia Ul Haq, M., Dubey, I, Dotiwala, K.N., Siddiqui, S.V., ... Nizamie, S.H., (2010). Effect of meta-cognitive training in the reduction of positive symptoms in schizophrenia. European Journal of Psychotherapy and Counseling, 12, 149-158.

Leucht, S., Arbter, D., Engel, R.R., Kissling, W., \& Davis, J.M. (2009). How effective are second-generation antipsychotic drugs? A meta-analysis of placebo-controlled trials. Molecular Psychiatry, 14, 429-447.

Lysaker, P.H., Olesek, K.L., Warman, D.B., Martin, J.M., Salzman, A.K., ... Dimaggio, G. (2011). Metacognition in schizophrenia: Correlates and stability of deficits in theory of mind and self-reflectivity. Psychotherapy Research, 190, 18-22.

McKay, R., Langdon, R., \& Coltheart, M. (2005). "Sleights of mind": Delusions, defences and self-deception. Cognitive Neuropsychiatry, 10, 305-326.

Moritz, S., Kerstan, A., Veckenstedt, A., Vitzthum, F., Schmidt, C., ... Woodward, T.S. (2011). Further evidence for the efficacy of a metacognitive group training in schizophrenia. Behavior Research and Therapy, 49, 151-157.

Moritz, S., Veckenstedt, R., Bohn, F., Hottenrott, B., Scheu, F., ... Roesch-Ely, D. (2013). Complementary group metacognitive training (MCT) reduces delusional ideation in schizophrenia. Schizophrenia Research, 151, 61-69.

Moritz, S., Veckenstedt, R., Randjbar, S., Vitzthum, F., \& Woodward, T.S. (2011). Antipsychotic treatment beyond antipsychotics: Metacognitive intervention for schizophrenia patients improve delusional symptoms. Psychological Medicine, 41, 1823-1832.

Moritz, S., Vitzthuma, F., Randjbar, S., \& Woodward, T.S (2010). Detecting and defusing cognitive traps: Metacognitive intervention in schizophrenia. Current Opinion in Psychiatry, 23, 561-569.

Moritz, S., Vitzthum, F., Veckenstedt, R., Leighton, L., Woodward, T.S., \& Hauschildt, M. (2012). Individualized metacognitive therapy program for psychosis (MCT+). Hamburg: VanHam Campus Press. 
Moritz, S., \& Woodward, T.S. (2006a). The contribution of metamemory deficits to schizophrenia. Journal of Abnormal Psychology, 115, 15-25.

Moritz, S., \& Woodward, T.S. (2006b). Metacognitive control over false memories: A key determinant of delusional thinking. Current Psychiatry Reports, 8, 184-190.

Moritz, S., \& Woodward, T.S. (2007). Metacognitive training in schizophrenia: From basic research to knowledge translation and intervention. Current Opinion in Psychiatry, 20, 619-625.

Naughton, M., Nulty, A., Abidin, Z., Abidin, Z., Davoren, M., O’Dwyer, S., \& Kennedy, H.G. (2012). Effects of group metacognitive training (MCT) on mental capacity and functioning in patients with psychosis in a secure forensic psychiatric hospital: A prospective-cohort waiting list controlled study. BMC Research Notes, 5, 302.

Pfammatter, M., Junghan, U.M., \& Brenner, H.D. (2006). Efficacy of psychological therapy in schizophrenia: Conclusions from meta-analyses. Schizophrenia Bulletin, 32(Supplement 1), s64-s80.

Pickup, G.J., \& Frith, C.D. (2001). Theory of mind impairments in schizophrenia: Symptomatology, severity and specificity. Psychological Medicine, 31, 207-220.

Sivec, H.J., \& Montesano, V.L. (2012). Cognitive Behavioral Therapy for Psychosis in clinical practice. Psychotherapy, 49, 258-270.

So, S.H., Garety, P.A., Peters, E.R., \& Kapur, S. (2010). Do antipsychotics improve reasoning biases? A review. Psychosomatic Medicine, 72, 681-693.

Speechley, W.J., Whitman, J.C., \& Woodward, T.S. (2010). The contribution of hypersalience to the "jumping to conclusions" bias associated with delusions in schizophrenia. Journal of Psychiatry and Neuroscience, 35, 7-17.

Tai, S., \& Turkington, D. (2009). The evolution of cognitive behavior therapy for schizophrenia: Current practice and recent developments. Schizophrenia Bulletin, 35, 865-873.

Wells, A. (2009). Metacognitive therapy for anxiety and depression. New York, NY: Guilford press.

Woodward, T.S., Moritz, S., Cuttler, C., \& Whitman, J.C. (2006). The contribution of a cognitive bias against disconfirmatory evidence (BADE) to delusions in schizophrenia. Journal of Clinical and Experimental Neuropsychology, 28, 605-617. 